\title{
Drug-resistant Streptococcus pyogenes: a case report of pyoderma and cellulitis
}

\author{
Barbara Pieretti, ${ }^{1}$ Benedetta Canovari, ${ }^{2}$ Marco Moretti, ${ }^{1}$ Carlo Pieretti, ${ }^{2}$ Enzo Pazzaglia ${ }^{1}$ \\ ${ }^{1}$ Clinical Pathology Laboratory and ${ }^{2}$ Infectious Diseases Department, Ospedali Riuniti Marche Nord General Hospital, \\ Fano (PU), Italy
}

\begin{abstract}
Summary
In the last years there has been worldwide an increase in the incidence of Streptococcus pyogenes infections and its sequelae. The purpose of the present study is to describe a clinical case of pyoderma and cellulitis by $S$. pyogenes with a pattern of resistance to penicillin, erythromycin and levofloxacin. Antimicrobial susceptibility was analysed by automated method of disk diffusion while E-test method was used to verify the minimum inhibitory concentration to penicillin, erythromycin and levofloxacin. Although penicillin remains the first-choice treatment for S. pyogenes infection, worldwide antibiotic resistance and the associated phenotypes are highly variable across countries. This case report strengthens the need for continued surveillance to obtain further insights into the forces governing resistance in $S$. pyogenes.
\end{abstract}

\section{Introduction}

Streptococcus pyogenes causes several infections as pharyngitis, pyoderma, cellulitis, necrotizing fasciitis, bacteraemia and

Correspondence: Barbara Pieretti, Department of Clinical Diagnostic Clinical Pathology Laboratory, Azienda Ospedaliera Ospedali Riuniti Marche Nord, S. Croce Hospital, Via Vittorio Veneto 2, 61032, Fano (PU) Italy.

Tel.: +39.0721.882379 - Fax: +39.0721.882304.

E-mail: barbara.pieretti@libero.it

Key words: Streptococcus pyogenes, antibiotics, erythromycin, penicillin, wound infection, resistance.

Contributions: BP and MM participated in conception and design of this report and were involved in drafting and revising the manuscript. BC, $\mathrm{CP}$ and EP participated in final revision of the manuscript.

Conflict of interest: the authors declare no potential conflict of interest.

Received for publication: 5 February 2017.

Revision received: 22 July 2017.

Accepted for publication: 28 July 2017.

CCopyright B. Pieretti et al., 2017

Licensee PAGEPress, Italy

Microbiologia Medica 2017; 32:6617

doi:10.4081/mm.2017.6617

This article is distributed under the terms of the Creative Commons Attribution Noncommercial License (by-nc 4.0) which permits any noncommercial use, distribution, and reproduction in any medium, provided the original author(s) and source are credited. pneumonia, besides post infectious sequelae like acute rheumatic fever.

Penicillin and its derivatives remains the drug of choice. However in patients allergic to penicillin, alternatives as macrolide and quinolones are prescribed (4).

Different resistance rates for $S$. pyogenes have been reported according to geographical location and investigators (5), proving how the spread of resistant strains not only to macrolides is increasing rapidly worldwide (3).

\section{Case Report}

A 43-year-old male, a fireman with no remarkable medical history, presented to the Emergency Department of our hospital on April 2016 secondary to an insidious onset of fever, persistent painful wounds on his left leg with swelling, redness, tenderness and difficulty walking around.

He was admitted to Infectious Diseases Department because of a suspected severe pyoderma and subsequently was diagnosed as having a cutaneous infection due to drug-resistant Streptococcus pyogenes.

He had a recent history of minor repeated traumas on his left leg related to his sport activities.

This patient does not smoke or drink alcohol with no allergies. He is a fit sportsman mostly consuming a vegetarian diet and practicing periodic fasting. The patient is not on chronic medication and was routinely vaccinated during his childhood and later against hepatitis A and B. Since 2001 at approximately annual intervals he has visited Asia (Indonesia, Sri Lanka), Australia and South America (Peru, Costa Rica) for surfing holidays.

During his most recent stay in Sri Lanka (from February to April 2016), while surfing, he endured several injuries caused by coral cuts on his feet and legs as well as a wound to the forepart of his left leg while he was playing football with local boys.

The patient returned to Italy in April with few persistent painful wounds on his left leg and foot and low-grade fever recurrent since the end of March 2016 for which he did not take any antibiotics due to his belief in alternative and related therapies. He applied a homemade aloe vera for several days and noted a progressive worsening of the wounds and of his clinical general conditions instead; spreading redness of the skin around purulent wounds, intense pain throughout his left lower limb, foot and ankle swelling and tenderness, high fever, malaise and Herpes labialis sore.

At admission, he was febrile but with normal vital signs; he reported intense pain on his left lower limb and walking difficulties. On the medial surface of the distal third of his left leg there 
were four ulcers which were intensely inflamed and discharging pus. Another less tender wound was seen at the base of the first finger of his left foot, medially. His left leg and foot were swollen, red and tender; a lymphangitic streak extended to his groin and there was inguinal lymphadenitis; herpes labialis being crusty; whitish exudate in retropharynx; modest signs of dehydration.

Laboratory test showed haemoglobin value of $15.0 \mathrm{~g} / \mathrm{dL}, \mathrm{MCV}$ $87.0 \mathrm{fl}$, platelets $215,000 / \mathrm{mm}^{3}$, white blood cell count of $11,100 / \mathrm{mm}^{3}$ (neutrophils $75 \%$, lymphocytes $14 \%$, monocytes $9 \%$ ), elevated inflammatory markers (ESR, CRP), fibrinogen and LDH; normality of the hepato-renal parameters and electrolytes.

Urine and blood cultures were negative, as well as an histological examination carried out on skin biopsy on the edge of the ulcers on purpose to rule out infectious agents as Leishmania and atypical mycobacteria (1). Both of the swab cultures from ulcers were positive for $S$. pyogenes resistant to macrolides, penicillin and levofloxacin.

S. pyogenes was suspected by Gram stain, colony morphology, catalase test, bacitracin sensitivity and positive reaction to serological identification done by Pastorex strep A (Bio-Rad Laboratories, Inc., Hercules, CA, USA).

The species identification (GP) and antimicrobial susceptibility testing (AST-ST01) were obtained by Vitek ${ }^{\circledR} 2$ system (bioMérieux, Marci-L'Etoile, France). The antimicrobial susceptibility categorisation was done accordingly to EUCAST 2016 clinical breakpoints.

This strain showed an antimicrobial profile of susceptibility to linezolid (MIC $\leq 2)$, tetracycline $(\mathrm{MIC} \leq 0.25)$, clindamycin $(\mathrm{MIC} \leq 0.25)$, trimethoprim/sulfamethoxazole $(\mathrm{MIC} \leq 10)$, vancomycin (MIC 1), and resistance to penicillin (MIC $\geq 8$ ), erythromycin (MIC $\geq 8$ ) and levofloxacin (MIC 4).

E-test strips (bioMérieux) for penicillin (MIC 32), erythromycin (MIC 8) and levofloxacin (MIC 16) was used to verify the resistance.

EUCAST version 7.1 bacteria breakpoint tables recommends for penicillin non-susceptible isolates to confirmed the identification and antimicrobial susceptibility test sending the strains to a reference laboratory because are rare or not yet reported, but unfortunately this was not possible because the strain was not stored.

Probably the phenotype isolate is type M (erythromycin resistant and clindamycin susceptible) (2).

During admission the patient was initially given intravenous amoxicillin/clavulanate for 2 days, with very modest benefit. Then, on the basis of the swabs results, he was treated with trimethoprim/sulfamethoxazole orally, with rapid defervescence and progressive improvement of his ulcers and inflammation of his left lower limb. The patient was discharged after 5 days from admission and continued treatment at home for 10 days.

\section{Discussion}

The isolation of drug-resistant S. pyogenes in a patient with no risk factor for nosocomial infections highlights the need to monitor the pattern of resistance of this important bacterial pathogen.

The streptococcus isolated from this patient was probably acquired in Sri Lanka, maybe playing football with the local boys or by coral cuts.

The isolation of a drug-resistant $S$. pyogenes in our case report paradoxally concerns a person, not only without any risk factor for nosocomial infections, but also with a reported peculiar distaste for antibiotics use, who was accustomed over several years to alternative remedies and phytotherapies.

With hindsight we should have performed a simple throat swab at the admission which maybe would have let us isolate the same strain from throat as well as from skin lesions.

\section{Conclusions}

Since incidence of erythromycin and penicillin resistance increased, restricted use of erythromycin and penicillin is advised to maintain required efficacy. Clindamycin is the drug of choice for penicillin resistant group A streptococci infections in children while vancomycin is best saved for life threatening conditions.

Wide-spread of resistance to erythromycin necessitates antibiotic guided therapy. Rational use of penicillin is a must not to lose this weapon in the fight against $S$. pyogenes.

The authors recommend storing strains belonging to microorganisms that show an unusual resistance strain to send them to reference laboratory (as Istituto Superiore di Sanità) to conduct more specific investigations such as $16 \mathrm{~S}$ rRNA gene sequencing region.

\section{References}

1. Doudi M, Setorki M, Narimani M. Bacterial superinfection in zoonotic cutaneous leishmaniasis. Med Sci Monit 2012;18:BR356-61.

2. Ibrahim SB, El-Sokkary RH, Elhewala AA, et al. Emerging resistance to erythromycin and penicillin among Streptococcus pyogenes Isolates in Zagazig, Egypt. Int J Curr Microbiol App Sci 2014;3:750-56.

3. Ray D, Sinha S, Saha S, et al. A preliminary sentinel surveillance report on antibiotics resistance trend of Streptococcus pyogenes in Kolkata region, India. Al Ameen J Med Sci 2010;3:146-51.

4. Sharma Y, Vishwanath S, Bairy I. Biotype and antibiotic resistance pattern of group A streptococci. Indian J Pathol Microbiol 2010;53:187-8.

5. Zhou W, Jiang YM, Wang HJ, et al. Erythromycin-resistant genes in group A - haemolytic Streptococci in Chengdu, South western China. Indian J Med Microbiol 2014;32:290-3. 